\title{
An Interpretation of Mechanical Properties of Bentonite as a Non-linear Elastic Material
}

\author{
Y. Takayama ${ }^{1}$, S. Tsurumi ${ }^{1}$, A. Iizuka ${ }^{1}$, K. Kawai ${ }^{1}$, S. Ohno ${ }^{2}$ \\ ${ }^{1}$ Kobe University, Japan; ${ }^{2}$ Kajima Corporation, Japan
}

\begin{abstract}
Bentonite is usually modeled as a geo-material with elasto-plastic isotropic compression/expansion and plastic dilatancy characteristics. In this paper, fundamental considerations of elasto-plastic constitutive modeling for fully saturated bentonite are investigated. According to uniaxial compression test data by Sasakura et al. (2003), where lateral earth pressure was measured, it was found that the hysteresis response between loading and unloading processes does not appear. It suggested that bentonite is an elastic material in which the swelling line corresponds to the normally consolidated line in the 'e-logp' relationship. Also, according to triaxial CU test results of bentonite, it was seen the effective mean stress remains almost unchanged during shearing. This implies that bentonite does not have dilatancy characteristics as defined by the critical state theory. Similar interpretation can be derived from the experimental results obtained from a series of triaxial CD tests by Cui et al. (2006).
\end{abstract}

Keywords: Bentonite, Elastic Material, Constitutive Modeling, Swelling Characteristic

\section{INTRODUCTION}

Bentonite is known to be a material that has remarkable expansion characteristics and very low permeability. Therefore, bentonite as a material, is expected to be useful as a buffer in the geological disposal of nuclear waste facility. As a result, its mechanical and mineralogical properties have been widely investigated. At nuclear disposal sites, bentonite is expected to play a role as a buffer, preventing leakage of radioactively polluted water by its abilities to self-seal and to mitigate stresses generated due to creep of base rock. Also, the geological disposal of nuclear material at such a facility needs to be stable and safe for an extremely long period of time. In order to address such problems, it is necessary to predict the long-term behavior of geological disposal at a nuclear waste facility and reliable numerical simulation techniques are required that can reliably address these issues. Constitutive modeling that describes the mechanical properties of a material is a key technique in such numerical simulations. Constitutive models for bentonite materials have been formulated phenomenologically based on the elasto-plastic response under loading and unloading processes and dilatancy characteristics during shearing, like other clayey materials. However, in the case of bentonite materials, we feel that the available experimental data to support constitutive modeling is not sufficient. Remarkable expansion characteristics on water absorption and very low permeability of bentonite make it difficult to interpret its fundamental mechanical behavior. The aim of this paper is to reconsider the fundamental mechanical behavior of bentonite materials based on recent experimental data and to discuss the theoretical formulation of constitutive modeling for bentonite materials.

\section{Isotropic compression and swelling characteristics of saturated bentonite materials}

Usually, isotropic compression and swelling characteristics are discussed based on the experimental data obtained from triaxial tests under a drained condition. However, in the case of bentonite materials, available experimental data on the compression and swelling characteristics obtained from triaxial tests under the drained condition are neither sufficient nor reliable. The primary data used to justify constitutive models have come from oedometer tests. Compression and swelling behavior of bentonite materials obtained from the oedometer tests are not directly applicable for discussing compression and swelling characteristics under isotropic confining pressure, because the lateral stress cannot be measured in the oedometer tests. If the lateral stress could reliably be estimated, more intensive discussion and more strict interpretation on the compression and swelling characteristics of bentonite materials would have been possible. In this paper, we cite experimental data on bentonite materials where lateral stress was measured with a special oedometer test apparatus by Sasakura et al. (2003). We also discuss the compression and swelling characteristics of bentonite materials under isotropic confining pressure, which are the fundamental mechanical properties for constitutive modeling.

\subsection{K0 compression and swelling test}

Sasakura et al. (2003) carried out one-dimensional compression and swelling tests for Kunigel V-1, which is typically made of sodium bentonite and is planned to be used in nuclear waste disposal sites in Japan. The authors tried to measure lateral stress under one-dimensional loading and unloading processes with a special oedometer test apparatus. Their experimental results are shown in Fig. 1. It was found that the coefficient of earth pressure at rest, $\mathrm{K} 0$, remains almost at 1.0 under the normal compression process and then increases with OCR under the unloaded swelling process. The relationship between $\mathrm{K} 0$ value and OCR is indicated in Fig. 2. In this figure, experimental data by Sasakura et al. (2003) is indicated by white circles and is compared to the empirical relation, Eq. (1) which relates $\mathrm{K} 0$-value and OCR through the evaluation parameter $\mathrm{m}$ as: 
$K_{0-O C} O=K_{0-N C} C R^{m}$

where $K_{0-N C}$ is the coefficient of earth pressure at rest under the normal compression process, $K_{0-O C}$ is the coefficient of earth pressure at rest under the unloaded swelling process, $O C R$ is the over-consolidation ratio defined by $\sigma_{v 0}^{\prime} / \sigma_{v}^{\prime}, \sigma_{v 0}^{\prime}$ is the pre-consolidation vertical stress, $\sigma_{v}^{\prime}$ is the current vertical stress and $m$ is the evaluation constant. According to Fig. 2, Eq. (1) with $m=0.6$, it seems to satisfactorily explain the experimental results of bentonite, Kunigel V-1. If tone employs Eq. (1) with 0.6 as the value of $m$, in converting the vertical stress into the isotropic confining pressure, fundamental mechanical properties on compression and swelling characteristics under isotropic confining pressure can be discussed using experimental data obtained from the oedometer test.

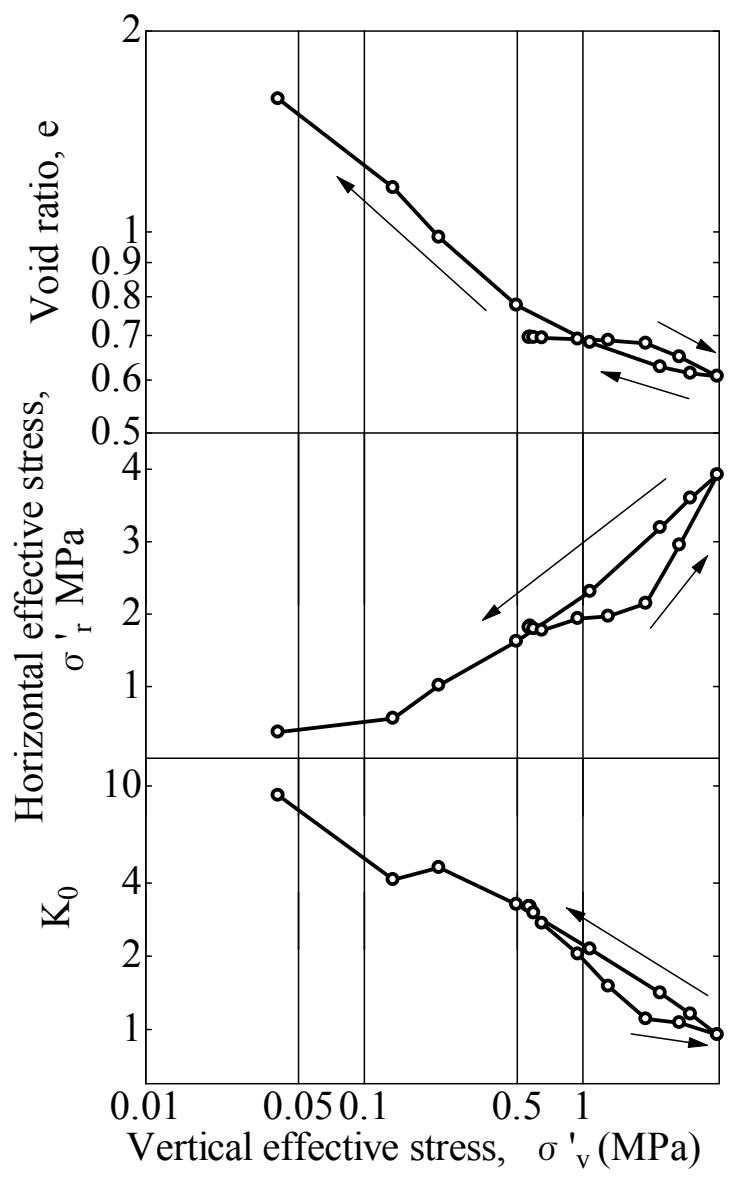

Fig. $1 \mathrm{~K}_{0}$ compression and swelling test result

\subsection{Isotropic deformation characteristics}

Fig. 3 shows one dimensional compression (consolidation) characteristics of Kunigel V1, obtained from oedometer tests by Sasakura et al. (2002) and Ishikawa et al. (1997). Normally, the consolidation lines by Sasakura et al. (2002) and Ishikawa et al. (1997) do not correspond to each other. This is because the nature of the bentonite slightly differs

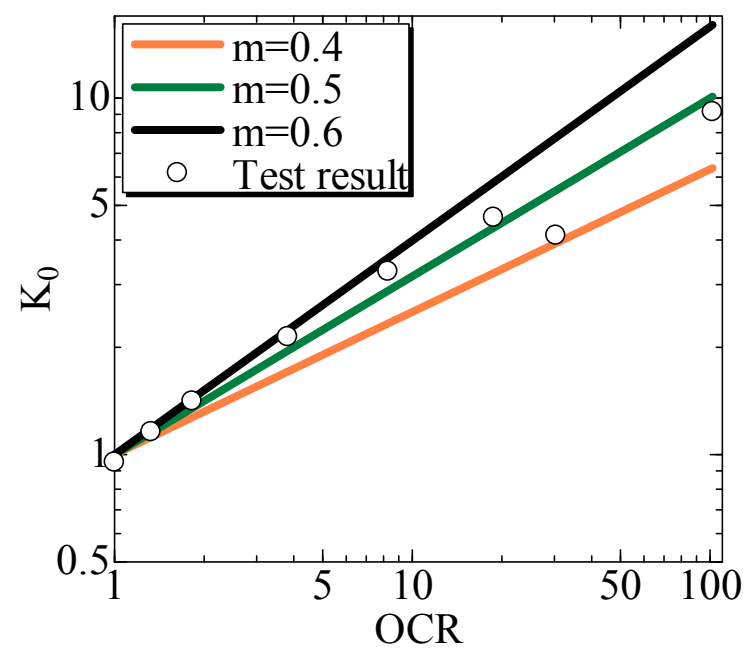

Fig. 2 Relationship between $\mathrm{K}_{0}$ value and OCR

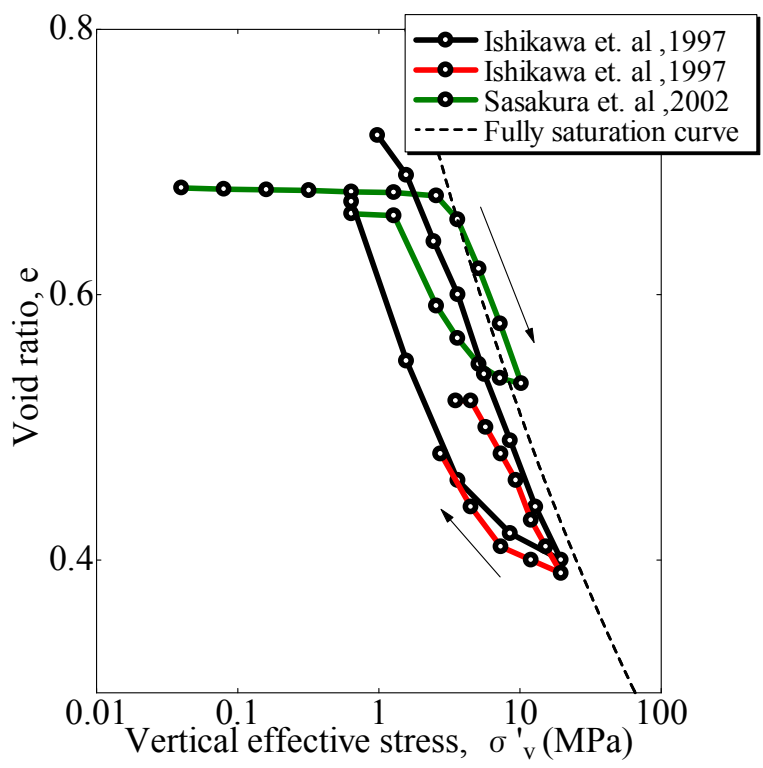

Fig. 3 One-dimensional compression (consolidation) characteristics of Kunigel V1

depending on the year it was produced and the content of the montmorillonite could be slightly different even in the same bentonite sample.

According to Kobayashi et al. (2007), one-dimensional normal consolidation characteristics of bentonite under the fully saturated state is uniquely expressed as:

$$
e=\lambda_{\rho} \ln \frac{\sigma_{v \max }^{\prime}}{\sigma_{v}^{\prime}} /\left(G_{s}-\lambda_{\rho} \ln \frac{\sigma_{v \max }^{\prime}}{\sigma_{v}^{\prime}}\right)
$$

where $\lambda_{\rho}$ is the material constant, $G_{s}$ is the specific gravity of soil particle and $\sigma_{v \max }^{\prime}$ is the reference and maximum vertical stress corresponding to the void ratio vanishing. This curve is called the full saturation curve. In the case of data by Sasakura et al. (2002), $\lambda_{\rho}=0.156$, $G_{s}=2.733, \sigma_{v \max }^{\prime}=3.73 \mathrm{GPa}$. 
The effective vertical stress can be converted into an effective mean stress using Eq. (1) as:

$p^{\prime}=\frac{\sigma_{v}^{\prime}+2 K_{0} \sigma_{v}^{\prime}}{3}$

where $p^{\prime}$ is the effective mean stress, $\sigma_{v}^{\prime}$ is the effective vertical stress and $K_{0}$ is the coefficient of earth pressure at rest. All data in Fig. 3 has been converted in terms of the effective mean stress using Eq. (3) and is depicted in Fig. 4 as a relation between the void ratio and the effective mean

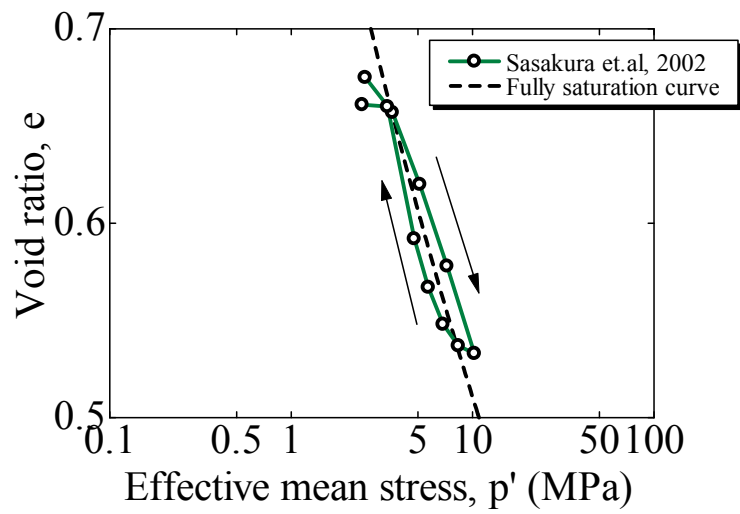

(A)

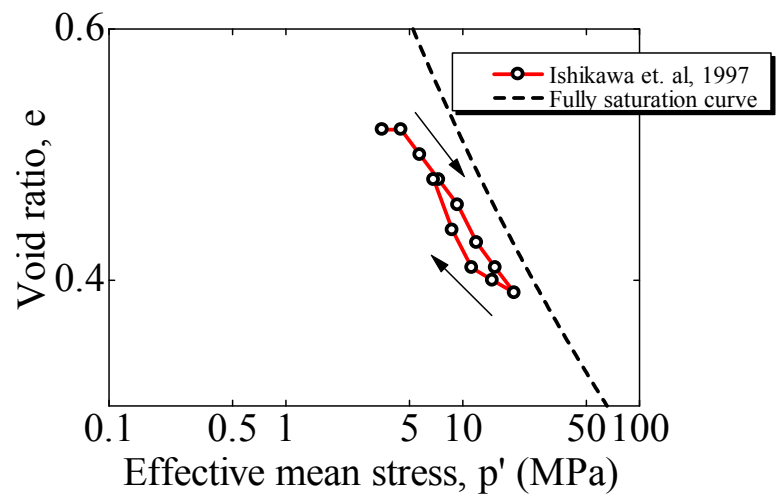

(B)

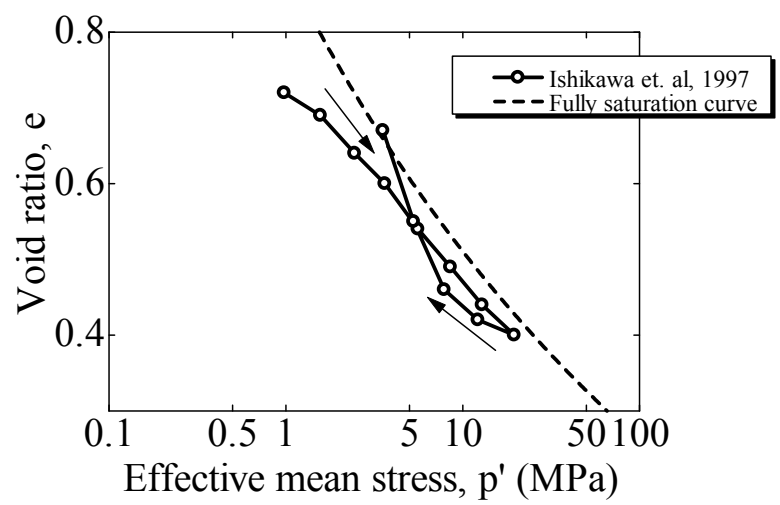

(C)

Fig. 4 Isotropic compression and swelling characteristics of saturated bentonite materials stress, in which $K_{0-N C}=1$ and $m=0.6$. It is found that the hysteresis-loop, between loading and unloading processes, disappears and the swelling line corresponds to the compression line in Fig. 4. If the compression and the swelling responses in Fig. 4 can be interpreted as the isotropic compression and the swelling characteristics of bentonite, the bentonite can be regarded as a material with no plastic deformation. In other words, it can be said that the bentonite is a non-linear elastic material.

\section{Dilatancy characteristics of saturated bentonite materials}

In this section, we examine the dilatancy characteristics of bentonite. Usually, dilatancy characteristics are examined by conducting triaxial $\mathrm{CD}$ tests in which the principal mean effective stress is maintained as a constant. However, in the case of bentonite materials, there is not sufficient reliable data being obtained from such tests. Therefore, we examine the dilatancy characteristic using the results of the triaxial $\mathrm{CU}$ test on bentonite.

\subsection{Triaxial CU test}

Results of triaxial CU test on a few kinds of bentonite are shown in Fig. 5-8. These figures show the relationship between effective mean stress and deviator stress. According to these test results, it can be seen that the effective mean stress is almost unchanged during shearing. Usually, the effective mean stress decreases with increasing shear stress because of negative dilatancy, when triaxial CU test on normally consolidated clay is conducted. It is therefore implied that the bentonite does not have dilatancy characteristics based on the critical state theory.

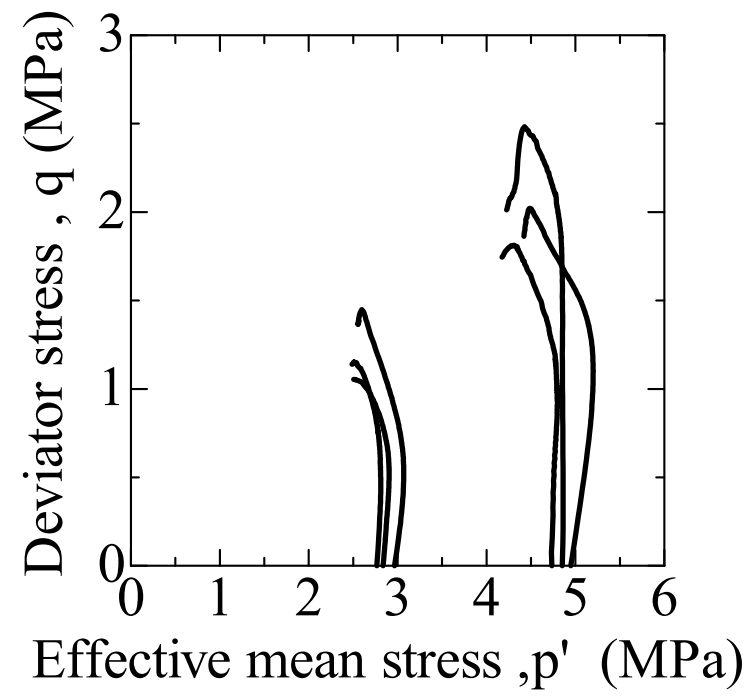

Fig. 5 Triaxial CU test result on sodium type of bentonite conducted by Sasakura et al. (2002) 


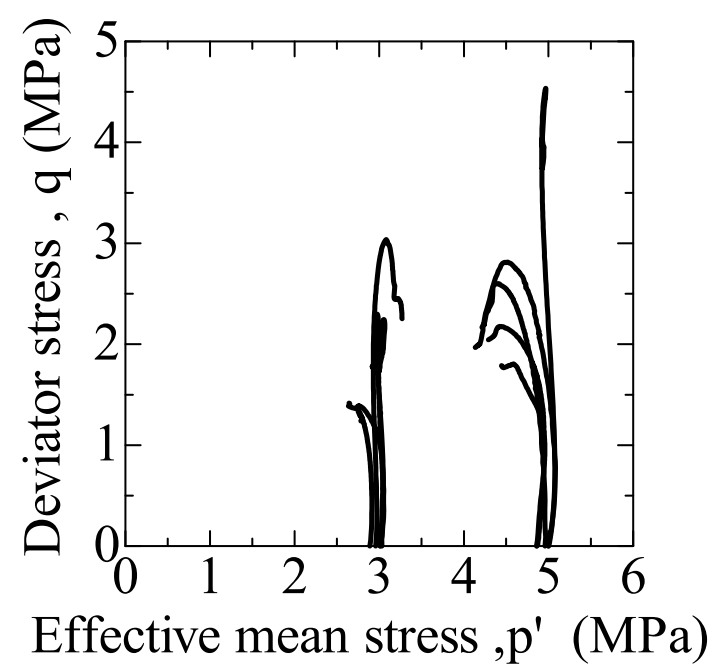

Fig. 6 Triaxial CU test result on calcium type of bentonite conducted by Sasakura et al. (2002, 2003)

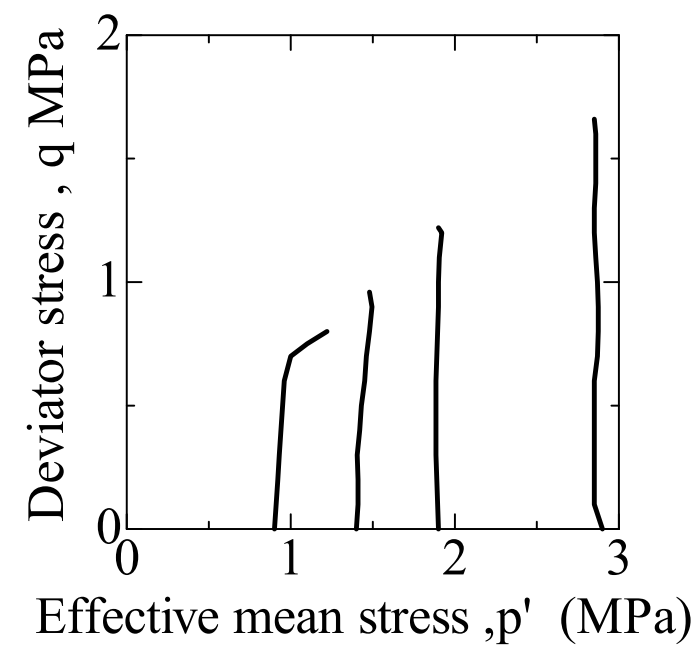

Fig. 7 Triaxial CU test result on OT-9607 conducted by Takaji et al. (1999)

\section{Swelling characteristic of saturated Bentonite}

An important mechanical property of bentonite is the swelling characteristic associated with the process of saturation from an unsaturated state. There are two kinds of tests for measuring swelling characteristics: the swelling deformation test and the swelling pressure test. Swelling deformation test reports deformations when the bentonite materials suck water to control the stress, and the swelling pressure test measures the swelling pressure when bentonite materials suck water to keep uniformity in the volume. Typically, these results are interpreted as a connection between pressure at saturation to dry density. However, the relationship between the results of this test and full saturation line are not perfectly correlated. Saturated bentonite is an elastic body, so it always lies on the full saturation line. Because of this, we estimate that the

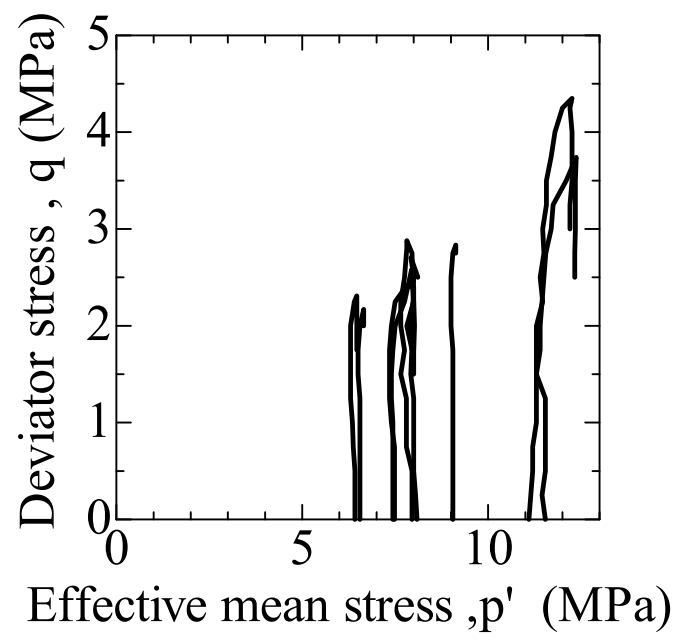

Fig. 8 Triaxial CU test result on MX-80 conducted by Ann et al. (2010)

final value of these swelling tests reach the full saturation line. Below, we examine the relationship between swelling characteristic and full saturation line, based on the results of this swelling test.

\subsection{Bentonite's swelling deformation test}

Cui (2006) conducted swelling deformation tests and isotropic unloading tests by controlling principal stress. These tests were performed on samples of compacted Kunigel V-1/Toyourasa mixture with Kunigel V-1 content of $50 \%$ in dry mass. In the tests, they set the initial dry density at three patterns: $1.69,1.54$ and $1.2 \mathrm{~g} / \mathrm{m} 3$; and then performed water absorption tests under hydrostatic stress, based on the condition that the tests maintain mean principal stress settled. The sample's initial values, initial dry density and initial water content ratio, and the value of mean effective principal stress under which the samples suck water in are shown in Table-1.

\begin{tabular}{|c|c|c|}
\multicolumn{2}{c}{ Table 1 Initial value } \\
\hline $\begin{array}{c}\rho_{\mathrm{d} 0} \\
\left(\mathrm{~g} / \mathrm{cm}^{3}\right)\end{array}$ & $\begin{array}{c}\mathrm{w}_{0} \\
(\%)\end{array}$ & $\begin{array}{c}\mathrm{p} \\
(\mathrm{kPa})\end{array}$ \\
\hline 1.69 & 18.7 & $49,98,147,196$ \\
\hline 1.54 & 18.7 & $19.6,49,88,196,294,392$ \\
\hline 1.2 & 19 & $49,98,196,392$ \\
\hline
\end{tabular}

Additional isotropic unloading tests were also performed, these reduce the load under $49 \mathrm{kPa}$ after the sample sucked water with mean principal stress set at $392 \mathrm{kPa}$. Fig. 9 shows these results: the relationship between mean effective principal stress and void ratio at the finish of retting and swelling of these three cases of initial dry density as well as the relationship between mean principal stress and void ratio when the test goes on isotropic unloading. In the case that it gave the sample some water in 
same mean stress, Fig. 9 shows that the void ratio takes the same value regardless of initial dry density. Furthermore, the value of unloading pass at isotropic unloading shows almost the same result as the swelling deformation test. This leads us to interpret that three words have the same meaning: full saturated line, normal consolidation line, and isotropic unloading line. Based on these results, one can conclude that the value, in the case of the swelling test, lies on the full-saturated line under saturated conditions. Next, swelling tests were conducted with different stress ratios $\mathrm{R}$ ( ) where the principal mean stress and the principal stress ratios were held constant. The initial values of the sample are: $1.69 \mathrm{~g} / \mathrm{m} 3$ for dry density and $18.7 \%$ as the initial water content ratio. We show the relationship between the void ratio and the mean principal stress at the end of soaking in Fig. 10. This figure shows that the void ratio and the mean stress exhibit a one-to-one correspondence regardless of the principal stress ratio. So, our examination that suggests saturated bentonite is a nonlinear elastic material without the characteristic of dilatancy coincides with the result of Cui's test.

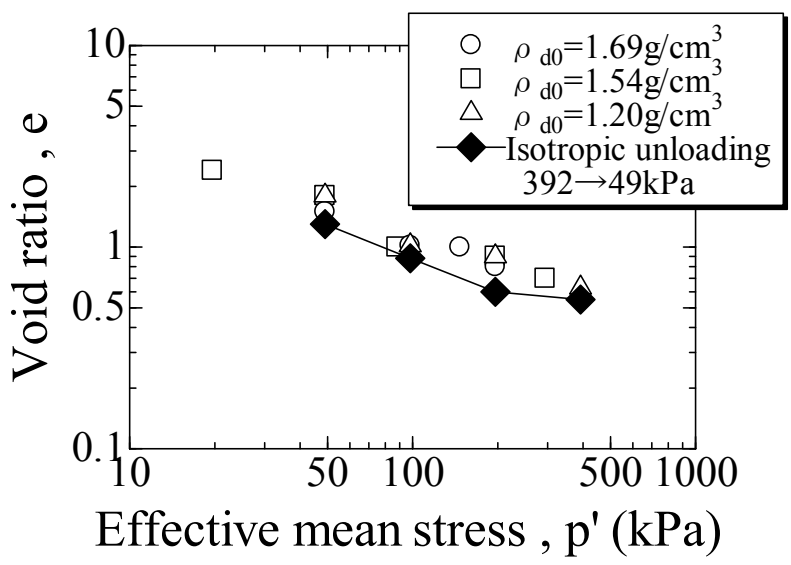

Fig. 9 Result of amount of swelling test

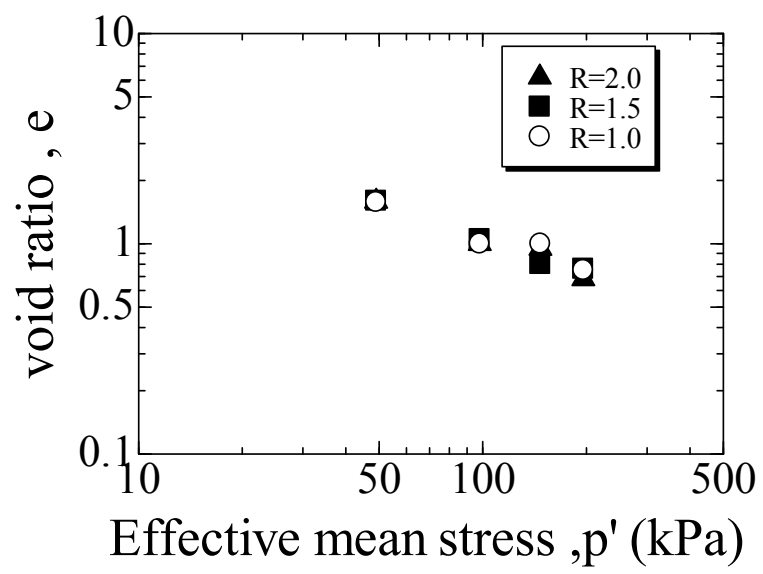

Fig. 10 Results of swelling test

\subsection{Swelling pressure test for bentonite}

A swelling pressure test, using equipment similar to that of the one-dimensional consolidation equipment, is a test that measures the counter force along the vertical direction caused by vertical soaking under settled volume. Fig. 11 shows the result of the swelling pressure test of Kunigel $\mathrm{V}-1$, and the relationship between the void ratio and the vertical swelling pressure at saturation. This figure conveys that when the void ratio is smaller, the swelling pressure becomes larger and the swelling pressure value varies in the same void ratio, but the value never crosses the full-saturated line. However, considering the results obtained so far, these separate variability values result from measuring only vertical swelling pressure. So, if we take account of lateral stress and reconsider it using mean stress, we consider that all values are on the fully saturated line.

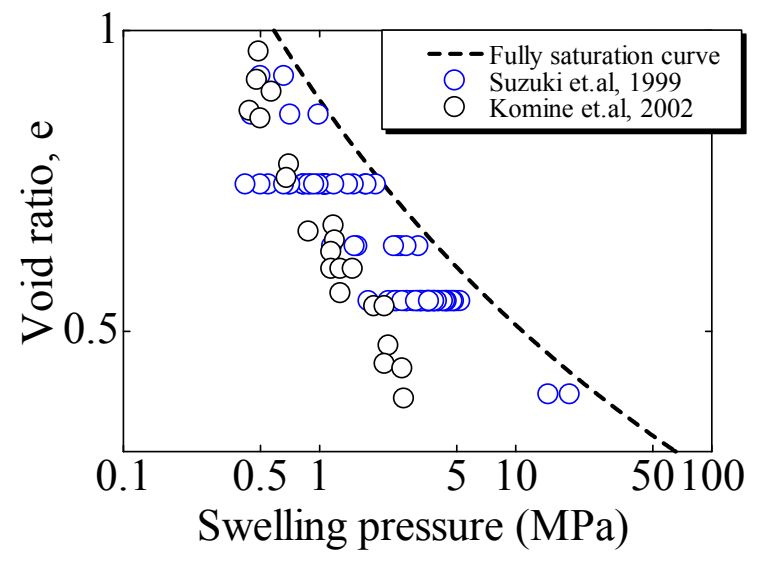

Fig. 11 the result of swelling pressure test

\section{Conclusion}

This study examines the mechanical characteristics of bentonite based on recent experimental data. The conclusions are summarized as follows:

(1) The swelling line at $S_{r}=1.0$ corresponds to the Normal Consolidation line. We also show that saturated bentonite does not have dilatancy characteristics. Therefore, saturated bentonite is a non-linear elastic material.

(2) When water is supplied and the unsaturated state is transformed to a saturated state, the final, or saturation, points achieve the full saturation curve.

In Fig. 12, the schematics of relationship between void ratio and the principal mean stress during loading, unloading and swelling are summarized.

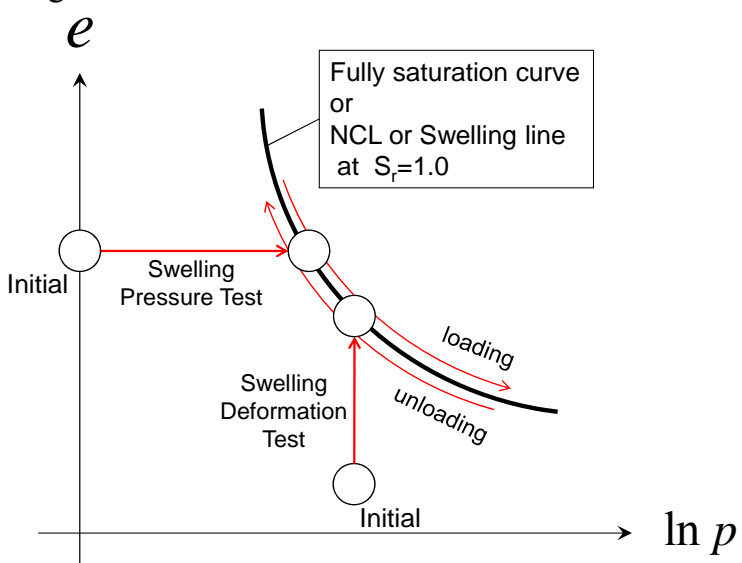

Fig. 12 Schematics of relationship between void ratio and the principal mean stress 


\section{REFERENCES}

[1] Sasakura, T., Kuroyanagi, M., Kobayshi, I., Okamoto, M. "Studies on mechanical behavior of bentonite for development of the constitutive model II", 2003, JNC TJ8400 2003-048. (in Japanese)

[2] Sasakura, T., Kuroyanagi, M., Okamoto, M. "Studies on mechanical behavior of bentonite for development of the constitutive model", 2002, JNC TJ8400-2002-025. (in Japanese)

[3] Ishikawa, H., Ishiguro, K., Namikawa, T., Sugano, T. "Consolidation Properties of Buffer Material" 1997, PNC TN8410 97-051. (in Japanese)

[4] Kobayshi, I., Toida, M., Sasakura, T. and Ohta, H. "Interpretation of compression/swelling behavior of compacted bentonite using constant water-content line and constant degree-of saturation line", 2007, JSCE Journal of Geotechnical Engineering, 63(4): 1065-1078. (in Japanese)

[5] Takaji, K., Suzuki, H. "Static Mechanical Properties of Buffer Material" 1999, JNC TN8400 99-041. (in Japanese)

[6] Ann Dueck, Lennart Börgesson, Lars-Erik Johannesson. "Stress-strain relation of bentonite at undrained shear" 2010, SKB Technical Report TR-10-32.
[7] Hongbin Cui, De'an Sun, Matsuoka, H. "Swelling characteristics of Sand-Bentonite mixtures under isotropic and anisotropic stress States" 2006, JSCE Journal of Geotechnical Engineering, 62(3): 657-666. (in Japanese)

[8] Suzuki, H. and Fujita, T. "Swelling Characteristics of Buffer Material" 1999, JNC TN8400 99-038. (in Japanese)

[9] Komine, H. and Ogata, N. "Swelling characteristics of sand-bentonite mixture and various kinds of bentonite", 2002, JSCE Journal of Geotechnical Engineering, 701(III-58): 373-385. (in Japanese)

International Journal of GEOMATE , Dec., 2012, Vol. 3, No. 2 (S1. No. 6), pp. 357-362.

MS No. 107 received June 15 2012, and reviewed under GEOMATE publication policies.

Copyright (C) 2012, International Journal of GEOMATE. All rights reserved, including the making of copies unless permission is obtained from the copyright proprietors. Pertinent discussion including authors' closure, if any, will be published in the Dec. 2013 if the discussion is received by June, 2013.

Corresponding Author: Y. Takayama 